\title{
Evidences in lifetimes of sequential $r$-out-of- $n$ systems and optimal sample size determination for Burr XII populations
}

\author{
Majid Hashempour * \\ Department of Statistics, School of Science, University of Hormozgan, Iran
}

(Received: 24 October 2016; Accepted: 16 May 2017)

\begin{abstract}
In this paper, the statistical evidences in lifetimes of dynamic $r$-out-of- $n$ systems, which are modelled by sequential order statistics (SOS), are studied. Weak and misleading evidences in SOS for hypotheses concerning the population parameters are derived in explicit expressions and their behaviours with respect to the model parameters are investigated in details. Optimal sample sizes are provided while a minimum desired level for the decisive and the correct probabilities is given. It is shown that the optimal sample size does not depend on some model parameters.
\end{abstract}

Keywords Burr model, Hypotheses testing, Likelihood ratio, Sequential order statistics

AMS 2010 subject classifications 62G30, 62F03, 62N05

DOI: $10.19139 /$ soic.v5i2.255

\section{Introduction}

Let $X_{1}, \cdots, X_{n}$ be independent and identically distributed (i.i.d.) random variables with a cumulative distribution function $(\mathrm{CDF})$, say $F$, and abbreviated by $X_{1}, \cdots, X_{n} \stackrel{i . i . d .}{\sim} F$. Denote in magnitude order of $X_{1}, \cdots, X_{n}$ by $X_{1: n} \leq \cdots \leq X_{n: n}$, which are called order statistics (OSs). Theory of OSs has been used widely in practice; See, e.g., David and Nagaraja [9] and references therein. In engineering system reliability analyses, lifetimes of $r$-outof- $n$ systems $(T)$ coincide to $X_{r: n}$. Here $X_{1}, \cdots, X_{n}$ stand for component lifetimes. If $X_{1}, \cdots, X_{n} \stackrel{i . i . d .}{\sim} F$, the OSs are used for describing the system lifetime.

Notice that failing a component does not change here the lifetimes of the surviving components. As mentioned by Cramer and Kamps [7], the failure of a component may result in a higher load on the surviving components and hence causes the lifetime distributions change. Examples of such phenomena include automobile industries, gas and oil transmission pipelines, etc. In these cases, the system lifetimes may be adequate to model by sequential(dynamic) order statistics (SOSs) as an extension of OSs. To see this, suppose that $F_{j}$, for $j=1, \cdots n$, denotes the $\mathrm{CDF}$ of the component lifetimes when $n-j+1$ components are working. The components begin to work independently at time $t=0$ with the CDF $F_{1}$. When at time $x_{1}$, the first component failure occurs, the remaining $n-1$ components are working with the CDF $F_{2}$. This process continues up to $n-r+1$ components with the CDF $F_{r}$ work until the $r$-th failure occurs at time $x_{r}$ and hence the whole system fails. The mentioned system is known as sequential r-out-of-n system and the system lifetime is then $r$-th component failure time, denoted by $X_{(r)}^{\star}$. In the literature, $\left(X_{(1)}^{\star}, \cdots, X_{(n)}^{\star}\right)$ is called SOSs; See, e.g., Cramer and Kamps [7]. Statistical inferences on the basis of SOSs have been considered in the literature. For example, Bedbur [3] obtained the

\footnotetext{
*Correspondence to: Majid Hashempour (Email: ma.hashempour@hormozgan.ac.ir). Department of Statistics, School of Science,
} University of Hormozgan , Bandar Abbas, Iran.

ISSN 2310-5070 (online) ISSN 2311-004X (print)

Copyright (C) 2017 International Academic Press 
uniformly most powerful unbiased test under a conditional proportional hazard rates (CPHR) model via a decisiontheoretic approach and Cramer and Kamps [8], Statistical inferences on the basis on one and two parameter exponential distributions. Let $\bar{F}_{j}(t)=\bar{F}_{0}^{\alpha_{j}}(t)$ for $j=1, \cdots, r$, where the underlying CDF $F_{0}(t)$ is a baseline DF. In this paper, the Burr XII distribution with DF

$$
F_{0}(x ; \sigma)=1-\left(1+x^{c}\right)^{-\theta}, \quad x>0, \quad \theta>0 .
$$

is considered.

The two-parameter Burr type XII distribution was first introduced by Burr (1942), and has gained special attention in the last two decades due to the importance of using it in practical situations. It has been applied in various areas of reliability studies and failure time data modelling [20]. The hazard rate function of the Burr CDF $F_{j}$, defined by $h_{j}(t)=f_{j}(t) / \bar{F}_{j}(t)$ for $t>0$ and $j=1, \cdots, n$, is proportional to the hazard rate function of the baseline CDF $F_{0}$, i.e. $h_{j}(t)=\alpha_{j} h_{0}(t)$. Statistical inference on the basis of SOS has been considered in literature; see, e.g, Beutner and Kamps [4], Cramer and Kamps [8], Esmailian and Doostparast [13], Hashempour and Doostparast [15], Schenk et al. [18], Shafay et al. [19] and references therein. Notice that for the special case $r=n$ and $\alpha_{1}=\cdots=\alpha_{n}$, the SOS reduce to ordinary order statistics based on a random sample from the CDF $F 1$. See also Table 1 of Ceramer and Kamps [8].

In this paper, we consider the problem of hypothesis testing for the Burr XII populations on the basis of multiple SOS samples under the CPHR model via a Bayesian approach. To do this, denote the available data by

$$
\mathbf{x}=\left[\left[x_{i j}\right]\right]_{i=1, \cdots, s, j=1, \cdots, r},
$$

where the $i$-th row of the matrix $\mathbf{x}$ in (2) denotes the SOS sample coming from the $i$-th population.

Some non-statistical scientists misuse statistical methods which lead to the misinterpretation of observations. For example, the decision-making paradigms since the work of Neyman and Pearson in the 1930s, have been formulated not in terms of interpreting data as evidence, but in terms of choosing between alternative course of actions. This lead to the current situation in which the Neyman-Pearson theory view common statistical procedures as decision-making tools, while much of statistical practice consists of using the same procedures for a different purpose, namely, interpreting data as evidence. In the Neyman-Pearson theory, a test of two hypotheses $H_{1}$ and $H_{2}$ is represented as a procedure for choosing between two actions. But in applications, when an optimal test chooses $H_{2}$, it is often taken to mean that data are evidence favoring $H_{2}$ over $H_{1}$. This interpretation can be quite wrong. For more details, see Blume [5, 6] and Royall [16, 17].

As mentioned above, the errors are usually quantitative, as when statistical evidence is judged to be weaker or stronger than it really is. So evidence is judged to support one hypothesis over another when the opposite is true. A key question is "when a certain hypothesis is preferred to others". In other words, when is it right to say that the observations are evidence in favour of one hypothesis vis-a-vis another? The answer to this fundamental question can be answered by Bayesian methods. But, the Bayesian methods need prior knowledge on the hypotheses. To avoid this problem, one may use non-informative priors or references analysis which are solely based on the observed data. In other words, one may consider the objective priors and then derive the posterior distributions of the hypotheses. Then the mentioned question can be answered by the posteriors; see, e.g., Berger [2] and references therein. This paper considers an alternative approach called evidential statistics which is also solely based on data. Following Royall [16], let $\lambda(>0)$ be a given data-based measure of support of $H_{1}$ against $H_{2}$. Large (Small) values of $\lambda$ can be interpreted as evidence given by data in favor of $H_{1}\left(H_{2}\right)$. The probabilities of observing strong misleading evidence under $H_{1}$ and $H_{2}$ are

$$
M_{1}=P\left(\lambda<\frac{1}{k} \mid H_{1} \text { is correct }\right),
$$

and

$$
M_{2}=P\left(\lambda>k \mid H_{2} \text { is correct }\right)
$$


respectively, where " $k$ " is a known constant greater than unity. The probability of weak evidence under $H_{i}(i=1,2)$ is

$$
W_{i}=P\left(\frac{1}{k} \leq \lambda \leq k \mid H_{i} \text { is correct }\right) .
$$

This paper considers evidences in independent multiple SOS samples given by (2) coming from homogeneous Burr populations under the above-mentioned CPHR model. Therefore, the rest of this paper is organized as follows: In Section 2, statistical evidences in SOS arising from the Burr XII populations are derived in explicit expressions and their behaviours with respect to the model parameters are studied in details. In Section 3, optimal sample sizes given a minimum desired level for the decisive and the correct probabilities are provided. Section 4 concludes.

\section{SOS-based evidences}

Let $X_{(1)}^{\star}, \cdots, X_{(r)}^{\star}$ be the first $r$ SOS. The joint probability density function of $\left(X_{(1)}^{\star}, \cdots, X_{(r)}^{\star}\right)$ is (Cramer and Kamps [8])

$$
f\left(y_{1}, \cdots, y_{r}\right)=B \prod_{j=1}^{r-1}\left[f_{j}\left(y_{j}\right)\left(\frac{\bar{F}_{j}\left(y_{j}\right)}{\bar{F}_{j+1}\left(y_{j}\right)}\right)^{n-j}\right] f_{r}\left(y_{r}\right) \bar{F}_{r}\left(y_{r}\right)^{n-r},
$$

for $y_{1}<y_{2}<\cdots<y_{r}, r=1, \cdots, n$, where $B=n ! /(n-1) !$ and $\bar{F}_{j}()=.1-F_{j}(),. j=1, \cdots, n$. From (6), the likelihood function (LF) of the data given by (2) reads

$$
L(\mathcal{F} ; \mathbf{x})=B^{s} \prod_{i=1}^{s}\left(\prod_{j=1}^{r-1}\left[f_{j}^{[i]}\left(x_{i j}\right)\left(\frac{\bar{F}_{j}^{[i]}\left(x_{i j}\right)}{\bar{F}_{j+1}^{[i]}\left(x_{i j}\right)}\right)^{n-j}\right] f_{r}^{[i]}\left(x_{i r}\right) \bar{F}_{r}^{[i]}\left(x_{i r}\right)^{n-r}\right)
$$

Under the CPHR modelling introduced in Section 1 and assuming that the baseline CDF in the $i$-th parent population $(i=1, \cdots, s)$ follows the Burr XII distribution with mean $\theta_{i}$, the LF of the available data is

$$
\begin{aligned}
L\left(\theta_{1}, \cdots, \theta_{s}, \boldsymbol{\alpha} ; \mathbf{x}\right) & =B^{s}\left(\prod_{j=1}^{r} \alpha_{j}\right)^{s}\left(\prod_{i=1}^{s} \theta_{i}\right)^{r}\left(\prod_{i=1}^{s} \prod_{j=1}^{r} x_{i j}^{c-1}\right) \\
& \times \prod_{i=1}^{s} \prod_{j=1}^{r}\left(1+x_{i j}^{c}\right)^{-\left(\theta_{i} m_{j}+1\right)} \\
& =B^{s}\left(\prod_{j=1}^{r} \alpha_{j}\right)^{s}\left(\prod_{i=1}^{s} \theta_{i}\right)^{r}\left(\prod_{i=1}^{s} \prod_{j=1}^{r} x_{i j}^{c-1}\right) \\
& \times \exp \left\{-\sum_{i=1}^{s} \sum_{j=1}^{r}\left(\theta_{i} m_{j}+1\right) \ln \left(1+x_{i j}^{c}\right)\right\},
\end{aligned}
$$

where $\boldsymbol{\alpha}=\left(\alpha_{1}, \cdots, \alpha_{r}\right)$ and $m_{j}=(n-j+1) \alpha_{j}-(n-j) \alpha_{j+1},(j=1, \cdots, r)$, with convention $\alpha_{r+1} \equiv 0$. When the baseline Burr XII populations are homogeneous i.e. $\theta_{1}=\cdots=\theta_{r}:=\theta$, the LF (8) simplifies to 


$$
\begin{aligned}
L(\theta, \boldsymbol{\alpha} ; \mathbf{x}) & =B^{s}\left(\prod_{j=1}^{r} \alpha_{j}\right)^{s}(c \theta)^{s r}\left(\prod_{i=1}^{s} \prod_{j=1}^{r} x_{i j}^{c-1}\right) \\
& \times \prod_{i=1}^{s} \prod_{j=1}^{r}\left(1+x_{i j}^{c}\right)^{-\left(\theta m_{j}+1\right)} \\
& =B^{s}\left(\prod_{j=1}^{r} \alpha_{j}\right)^{s}(c \theta)^{s r}\left(\prod_{i=1}^{s} \prod_{j=1}^{r} x_{i j}^{c-1}\right) \\
& \times \exp \left\{-\sum_{i=1}^{s} \sum_{j=1}^{r}\left(\theta m_{j}+1\right) \ln \left(1+x_{i j}^{c}\right)\right\} .
\end{aligned}
$$

\section{Remark 1}

One can show that $2 \theta \sum_{i=1}^{s} \sum_{j=1}^{r} \ln \left(1+X_{i ; j}^{c}\right) \sim \chi_{2 r s}^{2}$, where $\chi_{\nu}$ stands for the chi-square distribution with $\nu$ degrees of freedom.

Proof

Let $Y=\ln \left(1+X^{c}\right)$. The Jacobian transformation is

$$
J=\frac{1}{c} \exp \{y\}(\exp \{y\}-1)^{-\frac{1}{c}-1} .
$$

The probability density function of $Y$ is $f_{Y}(y)=\theta \exp \{\theta y\}$.

Then

$$
2 \theta \sum_{i=1}^{s} \sum_{j=1}^{r} \ln \left(1+X_{i ; j}^{c}\right) \sim \chi_{2 r s}^{2} .
$$

In the sequel, evidences in the available data (2) are derived for the simple hypotheses

$$
H_{1}: \theta=\theta_{1} \text { v.s } H_{2}: \theta=\theta_{2}
$$

where $\theta_{1}$ and $\theta_{2}$ are as known positive constants and $0<\theta_{1}<\theta_{2}$. Here, the likelihood ratio (LR) is implemented as a measure for evidence in data for the simple alternative hypotheses. To do this, Equation (9) gives the LR for the hypothesis $H_{1}$ against the alternative $H_{2}$ in (12) as

$$
\begin{aligned}
\lambda & =\left(\frac{\theta_{1}}{\theta_{2}}\right)^{s r} \prod_{i=1}^{s} \prod_{j=1}^{r}\left(1+x_{i j}^{c}\right)^{\left(\theta_{2}-\theta_{1}\right) m_{j}} \\
& =\left(\frac{\theta_{1}}{\theta_{2}}\right)^{s r} \exp \left\{\left(\theta_{2}-\theta_{1}\right) \sum_{i=1}^{s} \sum_{j=1}^{r} m_{j} \ln \left(1+x_{i j}^{c}\right)\right\}
\end{aligned}
$$


According to Remark 1 and Equations (3) and (13), the misleading probability is then derived as

$$
\begin{aligned}
M_{1} & =P\left(\left(\frac{\theta_{1}}{\theta_{2}}\right)^{s r} \prod_{i=1}^{s} \prod_{j=1}^{r}\left(1+x_{i j}^{c}\right)^{\left(\theta_{2}-\theta_{1}\right) m_{j}}<\frac{1}{k} \mid \theta=\theta_{1}\right) \\
& =P\left(\sum_{i=1}^{s} \sum_{j=1}^{r} m_{j} \ln \left(1+x_{i j}^{c}\right)<\frac{\ln \left(\frac{\left(\frac{\theta_{2}}{\theta_{1}}\right)^{s r}}{k}\right)}{\left(\theta_{2}-\theta_{1}\right)} \mid \theta=\theta_{1}\right) \\
& =P\left(2 \theta_{1} \sum_{i=1}^{s} \sum_{j=1}^{r} m_{j} \ln \left(1+x_{i j}^{c}\right)<\frac{2 \theta_{1}}{\left(\theta_{2}-\theta_{1}\right)} \ln \left(\frac{\left(\frac{\theta_{2}}{\theta_{1}}\right)^{s r}}{k}\right) \mid \theta=\theta_{1}\right) \\
& =F_{\chi^{2}{ }_{2 r s}}\left(\frac{2 \theta_{1}}{\left(\theta_{2}-\theta_{1}\right)} \ln \left(\frac{\left(\frac{\theta_{2}}{\theta_{1}}\right)^{s r}}{k}\right)\right)
\end{aligned}
$$

where $F_{\chi_{\nu}}$ is the CDF of the $\chi_{\nu}$-distribution and "ln" calls for the natural logarithm. Similar procedures yield the following proposition. The details are given in the appendix.

\section{Corollary 1}

Let $\tau=\theta_{2} / \theta_{1} \geq 1$. The misleading and weak evidences based on independent $s$ SOS samples from homogeneous Burr XII population under the CPHR model are

$$
\begin{gathered}
M_{1}=F_{\chi^{2}{ }_{2 r s}}\left(\frac{2 \ln \left(\tau^{s r} / k\right)}{\tau-1}\right), \\
M_{2}=1-F_{\chi^{2}{ }_{2 r s}}\left(\frac{2 \ln \left(k \tau^{s r}\right)}{1-\tau^{-1}}\right), \\
W_{1}=F_{\chi^{2}{ }_{2 r s}}\left(\frac{2 \ln \left(k \tau^{s r}\right)}{\tau-1}\right)-F_{\chi^{2}{ }_{2 r s}}\left(\frac{2 \ln \left(\tau^{s r} / k\right)}{\tau-1}\right),
\end{gathered}
$$

and

$$
W_{2}=F_{\chi^{2} 2 r s}\left(\frac{2 \ln \left(k \tau^{s r}\right)}{1-\tau^{-1}}\right)-F_{\chi^{2} 2 r s}\left(\frac{2 \ln \left(\tau^{s r} / k\right)}{1-\tau^{-1}}\right) .
$$

In particular, the probabilities in Equations (15)-(18) are free of the sample size $n$ and the parameter vector $\boldsymbol{\alpha}=\left(\alpha_{1}, \cdots, \alpha_{r}\right)$ of the assumed CPHR model.

An interesting topic in statistical evidence is determination of the global maximum of the misleading evidences. Here, the maximization $M_{1}$ in Equation (15) is equivalent to minimization $h(\tau)=\ln \left(\tau^{s r} / k\right) /\left(1-\tau^{-1}\right)$ with respect to $\tau \geq 1$. After some algebraic manipulations, one can see that the global minimum of $h(\tau)$ is derived by solving the non-linear equation $\partial h(\tau) / \partial \tau=0$, or equivalently $\ln (\tau)+1 / \tau=1-\ln (k) / s r$. Note that the function $h(\tau)$ is convex and therefore the solution of the mentioned equation is unique. Similar arguments for the misleading $M_{2}$ in Equation (16) imply the next proposition.

\section{Corollary 2}

Let $u(t)=1 / t+\ln (t)+\ln (k) /(s r)-1$, for $t \geq 1$. The points of global maximum of $M_{1}$ and $M_{2}$, as a function of $\tau$, are derived as the unique solutions of the non- linear equations $u(\tau)=0$ and $u(1 / \tau)=0$, respectively. 
Applying the well-known L' Hopital rule, one can prove that $\lim _{\tau \rightarrow+\infty} M_{i}=\lim _{\tau \rightarrow+\infty} W_{i}=0$. Notice that when $\sigma_{2}$ tends to infinity, the distance between the means of two populations will increase or will be increasing as much as possible. Thus, the probabilities of misleading and weak evidences tend to zero. So, even with inadequate(lack of data) one can make the decision about true hypothesis. Moreover, $\lim _{\tau \rightarrow 1^{+}} W_{i}=$ $\lim _{\tau \rightarrow 1^{+}}\left(1-M_{i}\right)=0$. Thus when $\sigma_{2}$ tends to $\sigma_{1}$, the distance between the means of two populations will decreasing as much as possible. So, $M_{1}$ and $M_{2}$ vanish and $W_{1}$ and $W_{2}$ tend to one. Hence, a decision cannot be taken based on the available data and one needs more SOS samples.

Consequently, we considered a general family of lifetime distributions. Al-Hussaini [1] proposed a general family of lifetime distributions of the form

$$
F(t ; \theta)=1-\exp \{-S(t ; \theta)\}, t>0,
$$

where the function $S(t ; \theta)$ is an increasing function in $t$ for all $\theta \in \Theta$ and $S(0 ; \theta) \equiv 0$.

Under the CPHR model and assuming that the baseline CDF of the baseline population belongs to the AlHussaini's family with the CDF (19), the LF in (7) simplifies to

$$
L(\theta, \boldsymbol{\alpha} ; \mathbf{x})=A^{s}\left(\prod_{j=1}^{r} \alpha_{j}\right)^{s}\left(\prod_{i=1}^{s} \prod_{j=1}^{r} \frac{\partial S\left(x_{i j} ; \theta\right)}{\partial x_{i j}}\right) \exp \left\{-\sum_{i=1}^{s} \sum_{j=1}^{r} m_{j} S\left(x_{i j} ; \theta\right)\right\},
$$

Now, we restrict ourselves to a subclass of the AL-Hussaini's family in which one may obtain explicit expressions for the MLE of the parameter vector $\theta$. More precisely, assume that

$$
F(t ; \theta)=1-\exp \{-w(\theta) h(t)\}, t>0,
$$

where $w($.$) is a non-negative function and h(t)$ is an increasing function and $h(0)=0$ and $h(t) \rightarrow+\infty$ as $t$ goes to infinity.

Applying (21), the LF (20) is reduced as

$$
L(\theta, \boldsymbol{\alpha} ; \mathbf{x})=\eta(\mathbf{x} ; \alpha) w(\theta)^{s r} \exp \{-w(\theta) \xi(\mathbf{x} ; \alpha)\},
$$

where $\xi(\mathbf{x} ; \boldsymbol{\alpha})=\sum_{i=1}^{s} \sum_{j=1}^{r} m_{j} h\left(x_{i j}\right)$ and

$$
\eta(\mathbf{x} ; \boldsymbol{\alpha})=B^{s}\left(\prod_{j=1}^{r} \alpha_{j}\right)^{s}\left(\prod_{i=1}^{s} \prod_{j=1}^{r} \frac{\partial h\left(x_{i j}\right)}{\partial x_{i j}}\right) .
$$

One can see from (22) that

$$
2 w(\theta) \xi(\mathbf{x} ; \alpha) \sim \chi_{2 r s}^{2},
$$

where $\chi_{\nu}^{2}$ calls for the chi-square distribution with $\nu$ degrees of freedom. Therefore, an equi-tail $100(1-\gamma) \%$ confidence interval for $w(\theta)$, with known parameter vector $\alpha$, is $\boldsymbol{\alpha}$ is known, is

$$
\left(\frac{\chi_{2 r s, \gamma / 2}^{2}}{2 \xi(\mathbf{x} ; \alpha)} \quad, \quad \frac{\chi_{2 r s, 1-\gamma / 2}^{2}}{2 \xi(\mathbf{x} ; \alpha)}\right)
$$

where $\chi_{\nu, \gamma}^{2}$ stands for the $\gamma$-th percentile of the $\chi_{\nu}^{2}$-distribution.

In the sequel, we consider evidences in the available data (2) for the problem of hypotheses testing

$$
H_{1}: w(\theta)=w\left(\theta_{1}\right) \text { v.s } H_{2}: w(\theta)=w\left(\theta_{2}\right)
$$

where $\theta_{1}$ and $\theta_{2}$ are known constants and $0<w\left(\theta_{1}\right)<w\left(\theta_{2}\right)$. To do this, Equations (13) and (22) yield the evidence for the hypothesis $H_{1}$ in favor of $H_{2}$ as

$$
\lambda_{w}=\frac{L\left(\theta_{1}, \boldsymbol{\alpha} ; \mathbf{x}\right)}{L\left(\theta_{2}, \boldsymbol{\alpha} ; \mathbf{x}\right)}=\left(\frac{w\left(\theta_{1}\right)}{w\left(\theta_{2}\right)}\right)^{s r} \exp \left\{\left(w\left(\theta_{2}\right)-w\left(\theta_{1}\right)\right) \xi(\mathbf{x} ; \alpha)\right\} .
$$


Corollary 3

The probabilities of misleading and weak evidences on the basis of $s$ independent SOS samples under the CPHR model with the baseline CDF (21) are

$$
\begin{aligned}
M_{1, w}= & F_{\chi^{2}{ }_{2 r s}}\left(\frac{2 w\left(\theta_{1}\right)}{\left(w\left(\theta_{2}\right)-w\left(\theta_{1}\right)\right)} \ln \left(\frac{\left(\frac{w\left(\theta_{2}\right)}{w\left(\theta_{1}\right)}\right)^{s r}}{k}\right)\right), \\
M_{2, w}= & 1-F_{\chi^{2}{ }_{2 r s}}\left(\frac{2 w\left(\theta_{2}\right)}{\left(w\left(\theta_{2}\right)-w\left(\theta_{1}\right)\right)} \ln \left(k\left(\frac{w\left(\theta_{2}\right)}{w\left(\theta_{1}\right)}\right)^{s r}\right)\right), \\
W_{1, w}= & F_{\chi^{2}{ }_{2 r s}}\left(\frac{2 w\left(\theta_{1}\right)}{\left(w\left(\theta_{2}\right)-w\left(\theta_{1}\right)\right)} \ln \left(k\left(\frac{w\left(\theta_{2}\right)}{w\left(\theta_{1}\right)}\right)^{s r}\right)\right) \\
& -F_{\chi^{2}{ }_{2 r s}}\left(\frac{2 w\left(\theta_{1}\right)}{\left(w\left(\theta_{2}\right)-w\left(\theta_{1}\right)\right)} \ln \left(\frac{\left(\frac{w\left(\theta_{2}\right)}{w\left(\theta_{1}\right)}\right)^{s r}}{k}\right)\right), \\
W_{2, w}= & F_{\chi^{2}{ }_{2 r s}}\left(\frac{2 w\left(\theta_{2}\right)}{\left(w\left(\theta_{2}\right)-w\left(\theta_{1}\right)\right)} \ln \left(k\left(\frac{w\left(\theta_{2}\right)}{w\left(\theta_{1}\right)}\right)^{s r}\right)\right) \\
& -F_{\chi^{2}{ }_{2 r s}}\left(\frac{2 w\left(\theta_{2}\right)}{\left(w\left(\theta_{2}\right)-w\left(\theta_{1}\right)\right)} \ln \left(\frac{\left(\frac{w\left(\theta_{2}\right)}{w\left(\theta_{1}\right)}\right)^{s r}}{k}\right)\right) .
\end{aligned}
$$

Proof

$$
\begin{aligned}
M_{1, w} & =P\left(\left(\frac{w\left(\theta_{1}\right)}{w\left(\theta_{2}\right)}\right)^{s r} \exp \left\{\left(w\left(\theta_{2}\right)-w\left(\theta_{1}\right)\right) \xi(\mathbf{x} ; \alpha)\right\}<\frac{1}{k} \mid w(\theta)=w\left(\theta_{1}\right)\right) \\
& =P\left(\exp \left\{\left(w\left(\theta_{2}\right)-w\left(\theta_{1}\right)\right) \xi(\mathbf{x} ; \alpha)\right\}<\frac{\left(\frac{w\left(\theta_{2}\right)}{w\left(\theta_{1}\right)}\right)^{s r}}{k} \mid w(\theta)=w\left(\theta_{1}\right)\right) \\
& \left.=P\left(\xi(\mathbf{x} ; \alpha)<\frac{\left.\ln \left(\frac{\left(\frac{w\left(\theta_{2}\right)}{w\left(\theta_{1}\right)}\right)}{k}\right) \mid w\left(\theta_{2}\right)-w\left(\theta_{1}\right)\right)}{(w r}\right) \mid w(\theta)=w\left(\theta_{1}\right)\right) \\
& =P\left(2 w\left(\theta_{1}\right) \xi(\mathbf{x} ; \alpha)<\frac{2 w\left(\theta_{1}\right)}{\left(w\left(\theta_{2}\right)-w\left(\theta_{1}\right)\right)} \ln \left(\frac{\left(\frac{w\left(\theta_{2}\right)}{w\left(\theta_{1}\right)}\right)^{s r}}{k}\right) \mid w(\theta)=w\left(\theta_{1}\right)\right) \\
& =P\left(\chi_{2 s r}^{2}<\frac{2 w\left(\theta_{1}\right)}{\left(w\left(\theta_{2}\right)-w\left(\theta_{1}\right)\right)} \ln \left(\frac{\left(\frac{w\left(\theta_{2}\right)}{w\left(\theta_{1}\right)}\right)}{k}\right)\right) \\
& =F_{\chi^{2}{ }_{2 r s}}\left(\frac{2 w\left(\theta_{1}\right)}{\left(w\left(\theta_{2}\right)-w\left(\theta_{1}\right)\right)} \ln \left(\frac{\left(\frac{w\left(\theta_{2}\right)}{w\left(\theta_{1}\right)}\right)}{k}\right)\right) .
\end{aligned}
$$

The decisive and correct evidences are, respectively, given by

$$
D_{1, w}=1-F_{\chi^{2}{ }_{2 r s}}\left(\frac{2 w\left(\theta_{1}\right)}{\left(w\left(\theta_{2}\right)-w\left(\theta_{1}\right)\right)} \ln \left(k\left(\frac{w\left(\theta_{2}\right)}{w\left(\theta_{1}\right)}\right)^{s r}\right)\right),
$$


and

$$
D_{2, w}=F_{\chi^{2}{ }_{2 r s}}\left(\frac{2 w\left(\theta_{2}\right)}{\left(w\left(\theta_{2}\right)-w\left(\theta_{1}\right)\right)} \ln \left(\frac{\left(\frac{w\left(\theta_{2}\right)}{w\left(\theta_{1}\right)}\right)^{s r}}{k}\right)\right) .
$$

\section{The optimal sample size}

Here, an optimal value for $s$ is obtained by minimizing $P^{\star}=\max \left\{M_{1}, M_{2}\right\}$ with a constraint on the $P_{D}=$ $\min \left\{D_{1}, D_{2}\right\}$, where $D_{1}$ and $D_{2}$ are called decisive and correct evidences, and are defined by $D_{1}=P(\lambda>$ $k \mid H_{1}$ is correct $)$ and $D_{2}=P\left(\lambda<1 / k \mid H_{2}\right.$ is correct $)$, respectively.

Notice that $D_{i}+M_{i}+W_{i}=1$, for $i=1,2$. Based on the available data in (2) and under the CPHR model, the decisive and correct evidences are given by

$$
D_{1}=1-F_{\chi^{2}{ }_{2 r s}}\left(\frac{2 \ln \left(k \tau^{s r}\right)}{\tau-1}\right)=1-F_{\chi^{2}{ }_{2 r s}}\left(\frac{2 \theta_{1}}{\left(\theta_{2}-\theta_{1}\right)} \ln \left(k\left(\frac{\theta_{2}}{\theta_{1}}\right)^{s r}\right)\right)
$$

and

$$
D_{2}=F_{\chi^{2}{ }_{2 r s}}\left(\frac{2 \ln \left(\tau^{s r} / k\right)}{1-\tau^{-1}}\right)=F_{\chi^{2}{ }_{2 r s}}\left(\frac{2 \theta_{2}}{\left(\theta_{2}-\theta_{1}\right)} \ln \left(\frac{\left(\frac{\theta_{2}}{\theta_{1}}\right)^{s r}}{k}\right)\right)
$$

respectively.

Proof

$$
\begin{aligned}
D_{1} & =P\left(\left(\frac{\theta_{1}}{\theta_{2}}\right)^{s r} \prod_{i=1}^{s} \prod_{j=1}^{r}\left(1+x_{i j}^{c}\right)^{\left(\theta_{2}-\theta_{1}\right) m_{j}}>k \mid \theta=\theta_{1}\right) \\
& =P\left(\sum_{i=1}^{s} \sum_{j=1}^{r} m_{j} \ln \left(1+x_{i j}^{c}\right)>\frac{\ln \left(k\left(\frac{\theta_{2}}{\theta_{1}}\right)^{s r}\right)}{\left(\theta_{2}-\theta_{1}\right)} \mid \theta=\theta_{1}\right) \\
& =P\left(2 \theta_{1} \sum_{i=1}^{s} \sum_{j=1}^{r} m_{j} \ln \left(1+x_{i j}^{c}\right)>\frac{2 \theta_{1}}{\left(\theta_{2}-\theta_{1}\right)} \ln \left(k\left(\frac{\theta_{2}}{\theta_{1}}\right)^{s r}\right) \mid \theta=\theta_{1}\right) \\
& =1-F_{\chi^{2}{ }_{2 r s}}\left(\frac{2 \theta_{1}}{\left(\theta_{2}-\theta_{1}\right)} \ln \left(k\left(\frac{\theta_{2}}{\theta_{1}}\right)^{s r}\right)\right)=1-F_{\chi^{2}{ }_{2 r s}}\left(\frac{2 \ln \left(k \tau^{s r}\right)}{\tau-1}\right),
\end{aligned}
$$


and

$$
\begin{aligned}
D_{2} & =P\left(\left(\frac{\theta_{1}}{\theta_{2}}\right)^{s r} \prod_{i=1}^{s} \prod_{j=1}^{r}\left(1+x_{i j}^{c}\right)^{\left(\theta_{2}-\theta_{1}\right) m_{j}}<\frac{1}{k} \mid \theta=\theta_{2}\right) \\
& =P\left(\sum_{i=1}^{s} \sum_{j=1}^{r} m_{j} \ln \left(1+x_{i j}^{c}\right)<\frac{\ln \left(\frac{\left(\frac{\theta_{2}}{\theta_{1}}\right)^{s r}}{k}\right)}{\left(\theta_{2}-\theta_{1}\right)} \mid \theta=\theta_{2}\right) \\
& =P\left(2 \theta_{2} \sum_{i=1}^{s} \sum_{j=1}^{r} m_{j} \ln \left(1+x_{i j}^{c}\right)<\frac{2 \theta_{2}}{\left(\theta_{2}-\theta_{1}\right)} \ln \left(\frac{\left(\frac{\theta_{2}}{\theta_{1}}\right)^{s r}}{k}\right) \mid \theta=\theta_{2}\right) \\
& =F_{\chi^{2}{ }_{2 r s}}\left(\frac{2 \theta_{2}}{\left(\theta_{2}-\theta_{1}\right)} \ln \left(\frac{\left(\frac{\theta_{2}}{\theta_{1}}\right)^{s r}}{k}\right)\right)=F_{\chi^{2}{ }_{2 r s}}\left(\frac{2 \ln \left(\frac{\tau^{s r}}{k}\right)}{1-\frac{1}{\tau}}\right) .
\end{aligned}
$$

Then the proof is complete.

Table 1. Optimal sample sizes for some selected values of $r, k, \tau$ and $\xi$.

\begin{tabular}{ccc|ccccc}
\hline & & & \multicolumn{5}{|c}{$\xi$} \\
\hline$r$ & $k$ & $\tau$ & 0.7 & 0.8 & 0.9 & 0.95 & 0.99 \\
\hline 3 & 3 & 2 & 4 & 5 & 8 & 11 & 19 \\
& & 5 & 1 & 1 & 2 & 3 & 4 \\
& & 8 & 1 & 1 & 1 & 2 & 3 \\
& 8 & 2 & 6 & 7 & 10 & 13 & 21 \\
& & 5 & 2 & 2 & 2 & 3 & 4 \\
& & 8 & 1 & 1 & 2 & 2 & 3 \\
\hline 5 & 3 & 2 & 2 & 3 & 5 & 7 & 11 \\
& & 5 & 1 & 1 & 1 & 2 & 3 \\
& & 8 & 1 & 1 & 1 & 1 & 2 \\
& 8 & 2 & 4 & 5 & 6 & 8 & 13 \\
& & 5 & 1 & 1 & 2 & 2 & 3 \\
& & 8 & 1 & 1 & 1 & 1 & 2 \\
\hline 15 & 3 & 2 & 1 & 1 & 2 & 3 & 4 \\
& & 5 & 1 & 1 & 1 & 1 & 1 \\
& & 8 & 1 & 1 & 1 & 1 & 1 \\
& 8 & 2 & 2 & 2 & 2 & 3 & 5 \\
& 5 & 1 & 1 & 1 & 1 & 1 \\
& & 8 & 1 & 1 & 1 & 1 & 1 \\
\hline
\end{tabular}

One can see that

- $P_{D}$ is increasing in $s$;

- $P_{D}$ is decreasing in $r$;

- $P_{D}$ is free of the sample size $(n)$.

As mentioned by De Santis [10], a sample size that guarantees $P_{D}$ reaches a desired level $\xi$, is often enough to bound the probabilities of weak and misleading evidences. Also, for chosen $\xi \in(0,1)$ and $k$, one then needs to derive

$$
s^{\star}=\min \left\{s \geq 1: P_{D} \geq \xi\right\} .
$$


Table 1 presents the values of the optimal sample size $s^{\star}$ given by (36) for some selected values of $n, r, k, \tau$ and $\xi$. According to Table 1, one can empirically see that the optimal value $s^{\star}$ is non-decreasing (non-increasing) in $\xi$ and $k$ (in $\tau$ and $r$ ), as we expected.

\section{Conclusions}

This paper considered statistical evidences in independent SOS arising from Burr XII populations. Weak and misleading evidences for simple hypotheses about the population parameter were derived in explicit expressions under the CPHR model. It was noticed that when $\sigma_{2}$ tends to infinity, the distance between the means of two populations would be increasing as much as possible. Thus, the probabilities of misleading and weak evidences tended to zero. So, even with restricted number of data we could make decision about true hypothesis. Also, when $\sigma_{2}$ was tending to $\sigma_{1}$, the distance between the means of two populations would be decreasing as much as possible. So, $M_{1}$ and $M_{2}$ would vanish and $W_{1}$ and $W_{2}$ would be tending one. Hence, one could not make decision based on the available data and needed more SOS samples. The probabilities in Equations (27)-(30) were free of the size $n$ of the system.

One can see that the optimal value $s^{\star}$ is non-decreasing in $\xi$ and $k$, and non-increasing in $\tau$ and $r$. The findings in the preceding sections hold for the cases when the vector $\boldsymbol{\alpha}$ in the CPHR model is unknown. Also, one can show that the optimal sample size $s^{\star}$ given by (36) is free of the vector $\boldsymbol{\alpha}$ and the sample size $n$. The results of this paper may be extended in some directions. For example, one may study statistical evidences for composite hypotheses. To do this, new measures of supports needs to be developed. Also, one may consider other lifetime distributions such as Pareto and log-normal distributions.

\section{REFERENCES}

1. E. K. AL-Hussaini, Predicting observable from a general class of distribution, Journal of Statistical Planning and Inference, vol. 79, pp. 79-91, 1999.

2. J. Berger, Statistical Decision Theory and Bayesian Analysis, Springer-Verlag, New York, 1985.

3. S. Bedbur, UMPU Tests based on Sequential order statistics, Journal of Statistical Planning and Inference, vol. 140, pp. 2520-2530, 2010.

4. E. Beutner and U. Kamps, Order restricted statistical inference for scale parameters based on sequential order statistics, Journal of Statistical Planning and Inference, vol. 139, pp. 2963-2969, 2009.

5. J. D. Blume, Likelihood methods for measuring statistical evidence, Statistics in Medicine, vol. 21, no. 17, pp. $2563-2599,2002$.

6. J. D. Blume, Likelihood and its evidential framework, In: Gabbay DM, Woods J (eds) Handbook of the philosophy of science: philosophy of statistics. North Holland, San Diego, pp. 493-511, 2011.

7. E. Cramer and U. Kamps, Sequential order statistics and k-out-of-n systems with sequentially adjusted failure rates, Annals of the Institute of Statistical Mathematics, vol. 48, no. 3, pp. 535-549, 1996.

8. E. Cramer and U. Kamps, Estimation with sequential order Statistics from exponential distributions, Annals of the Institute of Statistical Mathematics, vol,53, no.2, pp. 307-324, 2001a.

9. H. A. David and Nagaraja, Order Statistics, John Wiley \& Sons, Inc., 2003.

10. F. De Santis, Statistical evidence and sample size determination for Bayesian hypothesis testing, Journal of Statistical Planning and Inference, vol. 34, no. 124, pp. 121-144, 2004.

11. M. Doostparast and M. Emadi, Statistical evidence methodology for model acceptance based on record values, Journal of the Korean Statistical Society, vol. 35, no. 2, pp. 167-177, 2006.

12. M. Doostparast and M. Emadi, Evidential inference and optimal sample size determination on the basis of record values and record times under random sampling scheme, Statistical Methods and Applications, vol. 23, pp. 41-50, 2014.

13. M. Esmailian and M. Doostparast, Estimation based on sequential order statistics with random removals, Probability and Mathematical Statistics, vol. 34, no. 1, pp. 81-95, 2014.

14. U. Kamps, A concept of generalized order statistics, Journal of Statistical Planning and Inference, vol. 48, pp. 1-23, 1995.

15. M. Hashempour and M. Doostparast, Bayesian inference on multiply sequential order statistics from heterogeneous exponential populations with GLR test for homogeneity, Communications in Statistics-Theory and Methods, Doi.10.1080/03610926.2016.1175625, 2016.

16. R. Royall, Statistical Evidence: A Likelihood Paradigm, Chapman and Hall, New York, 1997.

17. R. Royall, On the probability of observing misleading statistical evidence, Journal of the American Statistical Association, vol. 95, pp. 760-780, 2000.

18. N. Schenk, M. Burkschat, E. Cramer and U. Kamps, Bayesian Estimation and Prediction with Multiply Type-II Censored Samples of Sequential Order statistics from one- and two-Parameter Exponential Distributions, Journal of Statistical Planning and Inference, vol. 141, pp. 1575-1587, 2011. 
19. A. R. Shafay, N. Balakrishnan, and K. S. Sultan, Two-sample Bayesian prediction for sequential order statistics from exponential distribution based on multiply Type-II censored samples, Journal of Statistical Computation and Simulation, vol. 84, no.3, pp. 526$544,2014$.

20. W. J. Zimmer, J. B. Keats, and F. K. Wang, The Burr XII distribution in reliability analysis, Journal of Quality Technology, vol. 30, pp. 386-394, 1998.

\section{A. Appendix}

Proof of Proposition 1: By Remark 1 and Equations (4), (5) and (13), we have

$$
\begin{aligned}
M_{2} & =P\left(\left(\frac{\theta_{1}}{\theta_{2}}\right)^{s r} \prod_{i=1}^{s} \prod_{j=1}^{r}\left(1+x_{i j}^{c}\right)^{\left(\theta_{2}-\theta_{1}\right) m_{j}}>k \mid \theta=\theta_{2}\right) \\
& =P\left(\sum_{i=1}^{s} \sum_{j=1}^{r} m_{j} \ln \left(1+x_{i j}^{c}\right)>\frac{\ln \left(k\left(\frac{\theta_{2}}{\theta_{1}}\right)^{s r}\right)}{\left(\theta_{2}-\theta_{1}\right)} \mid \theta=\theta_{2}\right) \\
& =P\left(2 \theta_{2} \sum_{i=1}^{s} \sum_{j=1}^{r} m_{j} \ln \left(1+x_{i j}^{c}\right)>\frac{2 \theta_{2}}{\left(\theta_{2}-\theta_{1}\right)} \ln \left(k\left(\frac{\theta_{2}}{\theta_{1}}\right)^{s r}\right) \mid \theta=\theta_{2}\right) \\
& =1-F_{\chi^{2}{ }_{2 r s}}\left(\frac{2 \theta_{2}}{\left(\theta_{2}-\theta_{1}\right)} \ln \left(k\left(\frac{\theta_{2}}{\theta_{1}}\right)^{s r}\right)\right)=1-F_{\chi^{2}{ }_{2 r s}}\left(\frac{2 \ln \left(k \tau^{s r}\right)}{1-\frac{1}{\tau}}\right) .
\end{aligned}
$$

Similarly

$$
\begin{aligned}
W_{1} & =P\left(\frac{1}{k}<\lambda<k \mid H_{1}\right)=F_{\chi^{2}{ }_{2 r s}}\left(\frac{2 \ln \left(k\left(\frac{\sigma_{2}}{\sigma_{1}}\right)^{s r}\right)}{1-\frac{\theta_{1}}{\theta_{2}}}\right)-F_{\chi_{2 r s}}\left(\frac{2 \ln \left(\frac{\left(\frac{\theta_{2}}{\theta_{1}}\right)^{s r}}{k}\right)}{1-\frac{\theta_{1}}{\theta_{2}}}\right) \\
& =F_{\chi^{2}{ }_{2 r s}}\left(\frac{2 \ln \left(k \tau^{s r}\right)}{\tau-1}\right)-F_{\chi^{2}{ }_{2 r s}}\left(\frac{2 \ln \left(\tau^{s r} / k\right)}{\tau-1}\right),
\end{aligned}
$$

and

$$
\begin{aligned}
W_{2} & =P\left(\frac{1}{k}<\lambda<k \mid H_{2}\right)=F_{\chi^{2}{ }_{2 r s}}\left(\frac{2 \ln \left(k\left(\frac{\theta_{2}}{\theta_{1}}\right)^{s r}\right)}{\frac{\theta_{2}}{\theta_{1}}-1}\right)-F_{\chi^{2}{ }_{2 r s}}\left(\frac{2 \ln \left(\frac{\left(\frac{\theta_{2}}{\theta_{1}}\right)^{s r}}{k}\right)}{\frac{\theta_{2}}{\theta_{1}}-1}\right) \\
& =F_{\chi^{2}{ }_{2 r s}}\left(\frac{2 \ln \left(k \tau^{s r}\right)}{1-\tau^{-1}}\right)-F_{\chi^{2}{ }_{2 r s}}\left(\frac{2 \ln \left(\tau^{s r} / k\right)}{1-\tau^{-1}}\right) .
\end{aligned}
$$

Then the proof is completed. 